\title{
METHODOLOGY FOR 3D ACQUISITION OF HIGHLY REFLECTIVE GOLDSMITHING ARTEFACTS
}

\author{
P. Hallot ${ }^{1 *}$, M. Gil ${ }^{2}$ \\ ${ }^{1}$ DIVA - Art, Archaeology and Heritage Research Unit, University of Liège, Liège Belgium - p.hallot@uliege.be \\ ${ }^{2}$ IRHiS, University of Lille, Lille, France-marc.gil@univ-lille.fr
}

\section{Commission II}

KEY WORDS: goldsmithing, cross-polarised light, photogrammetry, low cost, reflective objects, colour control

\begin{abstract}
:
The goldsmithing pieces conserved in museums are fragile objects whose accessibility for both research and mediation purposes lacks dematerialized systems for the safe handling of objects. The research presented is part of the e-thesaurus project whose main objective is to improve the accessibility of masterpieces of goldsmithing - produced during the medieval period in the northern regions of France and today's Belgium - both for research and mediation purposes. The methodology for 3D acquisition of objects with high levels of details and reflective properties must be further developed and affirmed in order provide controlled datasets that are suitable for researchers of the field and accurate for mediation application. The proposed project is the result of a collaboration between the IRHiS UMR CNRS of the University of Lille and the University of Liège. The proposed methodology leads to the 3D reconstruction of masterpieces of goldsmithing. The models developed serve as a basis for a mediation through holographic representation. They recently participated in an exhibition in the Treasure of the Cathedral of Liège museum.
\end{abstract}

\section{INTRODUCTION}

\section{1 e-thesaurus project}

The project e-thesaurus was born from a collaboration between the IRHiS UMR CNRS of the University of Lille and the University of Liège. The overall objective of the project is to improve the accessibility of masterpieces of goldsmithing produced during the medieval period in the northern regions of France and today's Belgium - for both research and mediation purposes. This paper presents the results of one of the technical phases of the project, which consists of the 3D modelling of several goldsmithing art-pieces. During the development of the project, the researchers focussed on a clear definition of the acquisition needs in relation to the objectives of the modelling, thanks to the large consortium gathering various fields: Goldsmithing specialists, art historians, curators, museums, geomatics researchers, religious arts experts and IT specialists. This broad panel helped to define precisely the demands of curators, researchers and museums mediation (Hallot and Gil, 2018). The working group's reflection to define the project's objectives led to two different fields of application. The first group of application requires a 3D model with the highest level of detail possible. The second group of request is intended for mediation purposes. The research consortium identified holographic representation as an innovative mediation device. The resolution of such a visualisation device is quite limited and a generalized model must be issued in order to be correctly visualized. The specification of scientific and mediation application have been discussed in (Hallot et al., 2019). Both models models are geo-referenced in the same coordinate system in order to easily switch from one to the other.

To develop the methodology of acquisition, we selected three objects that are conserved in the Hotel Sandelin Museum in the city of Saint-Omer (North of France). This museum holds in its collection the foot-cross of Saint Bertin Abbey (George, 2016), the reliquary cross of Clairmarais Abbey and a pyx (box to keep the consecrated Host), see Figure 1. These three objects were produced between the end of $12^{\text {th }}$ and the first decades of 13th century. They were chosen because of their geometric complexity and the large number of different materials they combine.
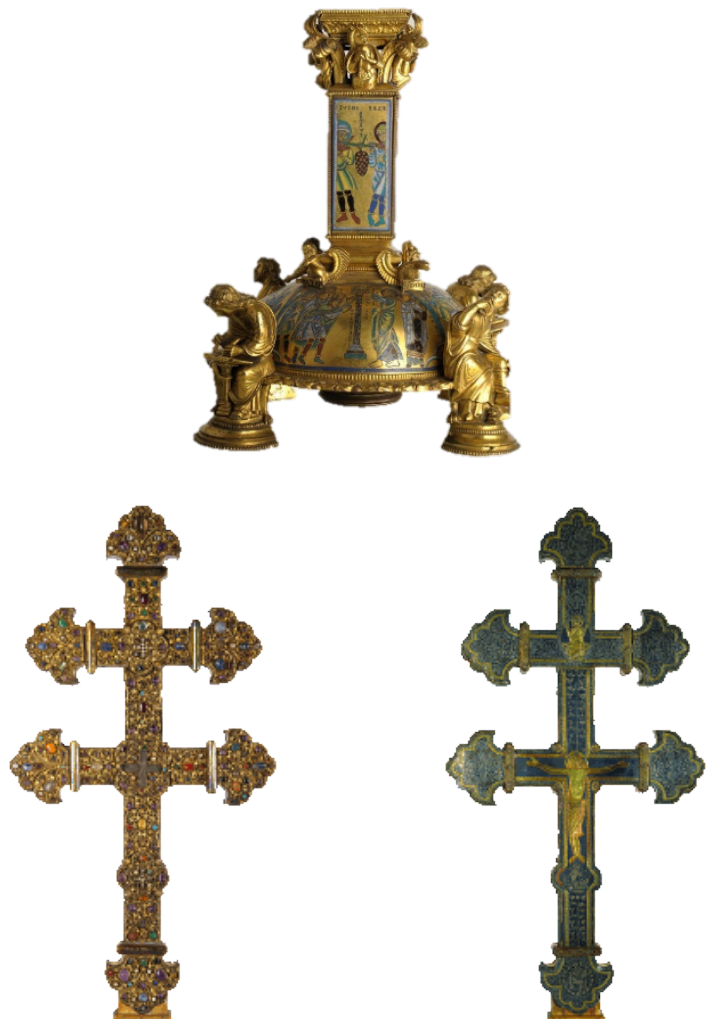

* Corresponding author 


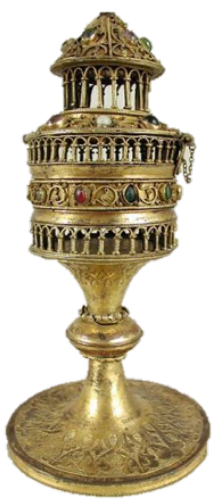

Figure 1. Foot-Cross of St Bertin, Cross of Clairmarais (both sides), Pyx

The specificities of the objects lead to the definition of the acquisition methodology. Four major characteristics lead to the selection of the photogrammetric approach in first intention. First, objects cannot be moved out of the museums. Indeed, these masterpieces must be handled with the greatest care, always with the help of the curator, in order to ensure the perfect state of conservation of the objects. This limits the possibility of using an acquisition methodology that requires heavy equipment or a laboratory. The second limitation is due to the materials that composes the objects. They are mainly made of metals (gold, silver) and glass. This type of materials is highly reflective and limits the use of active acquisition devices such as lidars or structured light scanners. In addition, such kind of devices are generally outside the range of costs that museums can achieve. In the framework of this project, this limitation must be taken into account because all the digitised are intended to develop and be produced by conservators themselves to feed the e-thesaurus database. The third limitation concerns the level detail that must be achieved by 3D models. Indeed, the selected pieces of art present a very high level of finesse, their precise chiselling, their openwork elements.. The level of targeted detail is a key factor in the choice of acquisition methodology. Finally, both models require a faithful texture rendering. In terms of mediation, the rendering of colour information ensures that the characteristic of the object is well communicated to the public without learning errors. The model dedicated to researchers can include several layers of colourimetric information based on different lightning conditions ranging from natural light to multispectral light. On the basis of the above elements, we naturally chose a photogrammetric approach to generate the 3D models of the selected objects. Depending on the quality of the results or technical developments, other methodologies could be tested.

\section{RELATED WORKS}

The acquisition of artefacts based on the application of the photogrammetric process has been known for several years. Initially developed for larger areas, aerial photogrammetry has evolved to close range photogrammetry for smaller objects and restricted areas (Luhmann et al., 2007). The techniques allow versatile acquisition in a wide range of situations. However, photogrammetry, as a passive acquisition technique, requires an external light source. Variable lighting conditions can lead to model adjustment difficulties, especially during the feature detection process and, therefore, during automatic image comparison. Reflection points cause errors in reconstruction and create artefacts in final models. The variability of the final texture applied to the 3D models is another limitation due to the variable lightning condition. Indeed, depending on the temperature of the light, the same object can be rendered in two completely different ways (Nicolae et al., 2014). The necessary basis for a good reconstruction is a good shot, therefore a good lighting condition. The acquisition of good images for reflective objects is not limited to photogrammetric application or heritage documentation. From medical applications (Edwards, 2011) to palaeontology (Crabb, 2001), the cross-polarized lightning technique is used to avoid reflection on bright objects. These elements tend to reflect incident light in all directions, giving the acquired image a "glow" (Edwards, 2011). The polarization of light is obtained by using a polarized filter that only allows the light wave in one direction, see Figure 2.

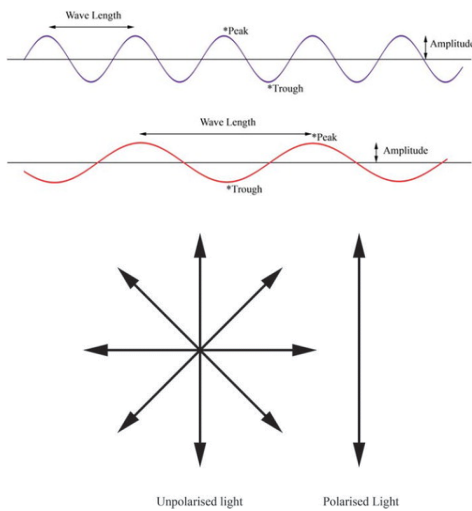

Figure 2. Upper figure shows a light wave representation considering two different wavelengths. The bottom figure shows a cross-sectional view of the light waves: left based on un-polarised light waves; right polarised light wave. From (Edwards, 2011).

This principle is applied during the acquisition process with two filters. One is placed at the light source (or the lights) and the other just in front of the acquisition device. Figure 3 shows the work plan that was been tested by (Noya et al., 2015).

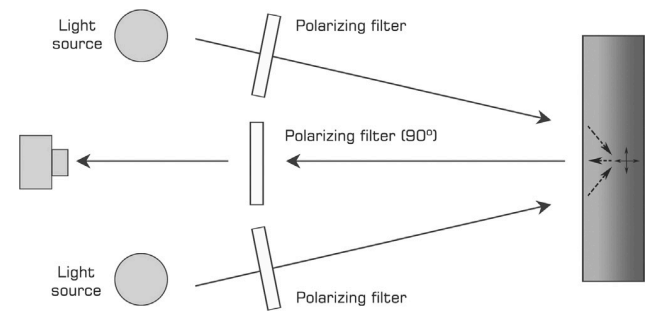

Figure 3. Installation for polarised light acquisition, from (Noya et al., 2015).

When cross-polarized images are used in photogrammetry, they tend to improve photogrammetric reconstruction and measurement on reflective surfaces (Wells et al., 2005). Conen et al. show that image matching is significantly improved when polarized light conditions are used (Conen et al., 2018). However, they showed that the use of polarized light changes the parameters of the indoor camera. A calibration of the camera is therefore necessary to change the lighting conditions. Nicolae et al. tend to use polarization for problematic artefacts where light reflection causes problems. Polarized light is also used when textures are too homogeneous and limits the detection of elements that cause orientation errors (Nicolae et al., 2014). Abate combine this technique with several wave-lengths lights (UVs, IR ...) to document the restoration of the paint with change detection algorithms. In this case, polarized light helps to 
compose a complete set of information that will be used in the change detection process (Abate, 2019).

\section{ACQUISITION METHODOLOGY}

The acquisition methodology is developed in two phases. The first approach uses natural light to reconstruct objects. We first focused on the cross of Saint Bertin because of its relative ease of acquisition. We believe that the reflection of the object is limited enough to test an acquisition inside a light tent and flash snoods to avoid reflections. This will provide a basis for validation for the reconstruction of polarized light. The modelled objects require the construction of supports strong enough to support their weight and high enough to have an open view of the bottom of the object, in order to acquire the bottom of the structure. The acquisition of the complete structure of the object is something just as important for the medieval art specialist to understand the construction of such objects. At the end, they would be able to measure the width of the elements. This requirement leads us to propose two models (one from the outside and one from the inside) geo-referenced in the same system. The developed supports will provide common reference points to ensure the quality of the settings and limit misinterpretation based on geometric inaccuracy.

From the first model, we have designed supports on which reference scales can be positioned. The material of the support must be compatible with the preservation of the object. The rigid foam has been chosen by the conservators. Printed plastics or wooden structures were not compatible with goldsmith's items. The support was not rigid enough to use controlled point systems. Then, we decide to multiply the scale bars of different sizes and label each target to link the inside and outside models.

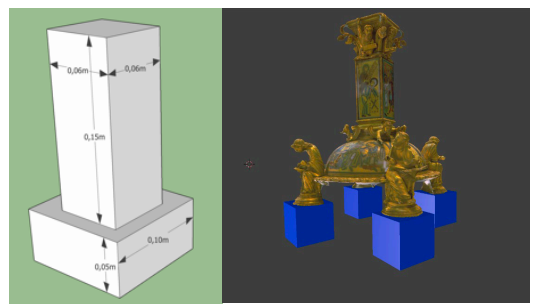

Figure 4. Design of an acquisition support based on a first raw model acquired through a protective glass.

\subsection{Natural light acquisition}

The first acquisition round is based on diffuse natural light. The objects are placed in a diffusion tent and the flashes are mounted with snoods. To avoid any disturbance of the external light, we preferred to use flashes with a neutral distortion filter mounted on the camera. This helps to better control exposure and prevent the flash effect from being felt.

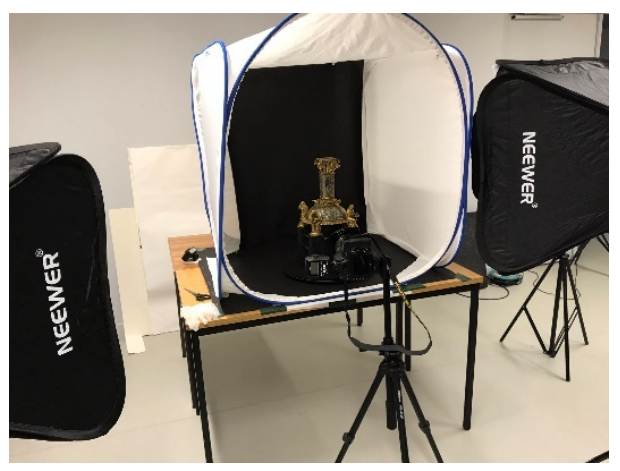

Figure 5. Natural light acquisition setup. Objects are placed in tents and flashed are mounted with snoods.

The model was acquired with a Nikon D70 mounted with a $110 \mathrm{~mm}$ Nikkor Macro lens, some tests were performed with a $50 \mathrm{~mm}$ Nikkor. In each case, we targeted a ground sampling distance of $0.25 \mathrm{~mm}$. In all cases, the overlap between the images was forced to the maximum in order to limit the reflection effect and smooth the reflection when adjusting the colour mixture. The image colours and light temperature were calibrated using Datacolour's SpyderCheckr24. For each model we acquired around 2500 pictures.
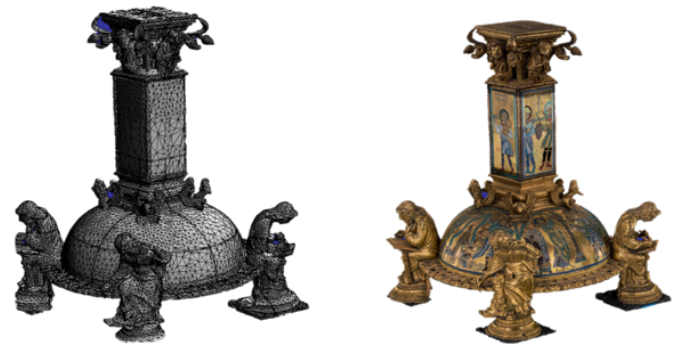

Figure 6. 3D model (with and without texture) obtained in natural lighting condition.

Despite the good visual results of the reconstruction, some texture details remain unclear. We explain this limitation by using a macro lens with a very limited depth of field. To avoid reflections, it was not possible to force the lighting too hard to reach a greater depth of field. In addition, the colour mixing performed by Context Capture software created artefacts when mixing white dots on some photos.

\subsection{Cross-polarised light acquisition}

The second acquisition round is carried out in cross-polarised light condition. We choose to develop an affordable system in order to capture the images. We setup two lighting systems. The first one with permanent light obtained from a led panel, the second one with flashes. For both, in order to polarize the lights a filter has to be placed in front of the lightning device. The orientation of the polarizing filter must be controlled to ensure that the light is polarised in the correct direction (see figure 7). The complete set of permanent light is estimated to cost less than 250 EUR. 


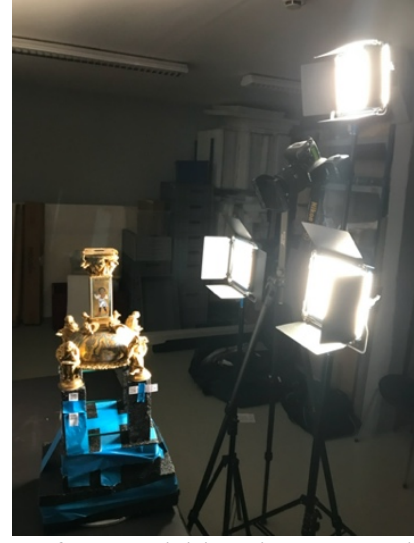

Figure 7. Setup for acquisition in cross-polarised lightning condition. A linear polarizing filter is placed in front of the led panels. A rotative linear filter is mounted on the lens in order to active or inactivate the polarization.

In this case, the position of the permanent lighting allows better control of the lighting position and eliminates more reflections. Figure 8 shows the difference between polarized light and normal light. The camera is mounted with a NiSi linear polarizing filter mounted on a rotating device. This allows you to take pictures with active or non-active cross-polarization. As can be seen in Figure 8, the reflection is completely eliminated on the right image (with cross-polarized light). We notice that the results show less reflection when the position of the LED panels is parallel to the camera filter, i.e. the sensor. A very incidental flash will create a reflection that is very difficult to eliminate. Finally, all the acquisitions were made without any external light that causes variations in the images. Following this methodology, a total of 2618 images were acquired with a Nikon D70 (full frame) and two 85 and $120 \mathrm{~mm}$ focal lengths. We chose this approach of two different focal lengths in order to maximize the depth of field according to the shape of the object and to reach the target ground sampling distance. A colour chart was acquired under both lighting conditions. The use of these images will be explained in the next section.
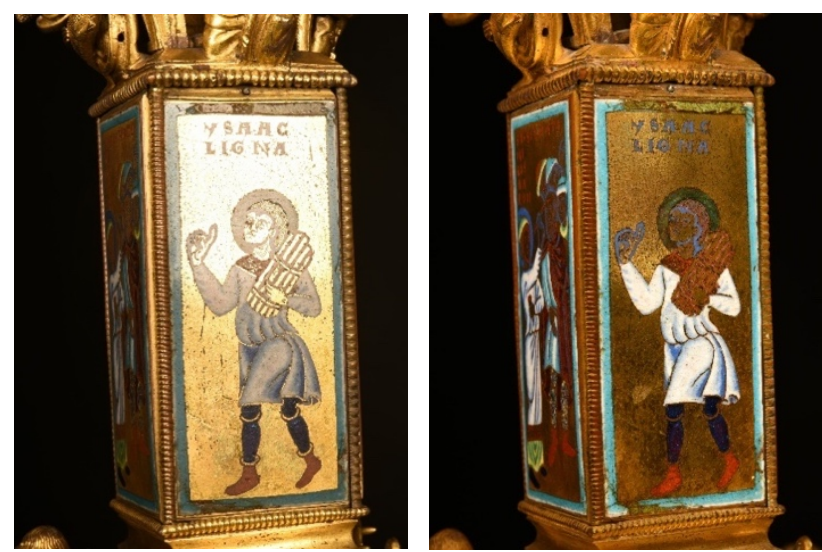

Figure 8. Left, picture acquired in normal light condition. Right, same scene acquired with cross-polarised light condition.

However, a major disadvantage appears in such a state of illumination, especially when it comes to goldsmith's materials. Indeed, as shown in Figure 8, the colours obtained vary significantly from exposure to natural light. This limitation is problematic for both scientific purposes and mediation.
Mediation requires a colouring as close as possible to reality. The scientific community can benefit from the use of a polarized light model to detect details that are not necessarily visible in natural light, but must always be combined with a natural colour representation.

\section{MODEL ADJUSTMENT}

\subsection{Colour calibration}

To avoid strong colour changes in the model's textures, we have corrected the colours according to the visilibty of a colour chart. The colour temperature of the light source does not change from one lightning situation to another. They both remain at 3700 Kelvin with the led pannel used for the acquisition. Figure 7 shows the chart in both lightning situations. The historgrams of the pictures does not visually show a significant change of the colour representation itself (the hue). However, the contrast of the images increases with the polarized light. This is confirmed by comparing the two imags histograms calculated in RGB colour representation (figure 10). The images were acquired with the same configuration, exposure and aperture.
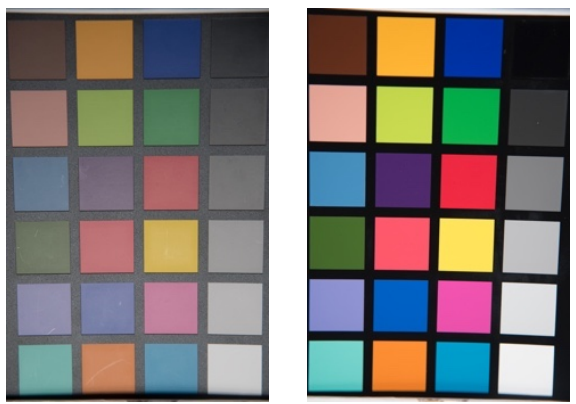

Figure 9. Left: colour chart acquired through natural light condition. Right: chart acquire with cross-polarised light condition.
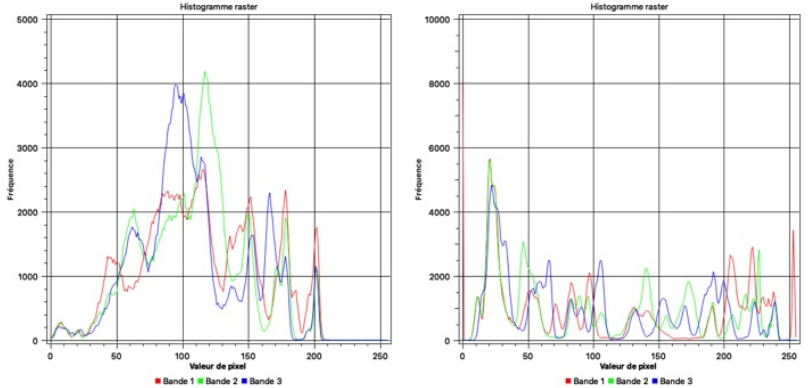

Figure 10. Colour histograms computed from the calibration chart in two different lighting situation. Natural light condition (left), Cross-polarised light (right).

Depending on the objects acquired, we notice that the colour variation is more affected by the variation of materials than a common shift of the entire colour histogram or a distortion of some colours. However, we have calculated a colour calibration scheme based on the colour chart. We applied calibration to each image according to the light situation. In fact, we calculated a colour adjustment for each light condition. The resulting models were visualized by art history experts to validate the representation. This external expertise is very important in order to provide accurate representation for mediation. The control process based on expertise ensures a correct dissemination of the goldsmith's trade. 


\subsection{Geometric results}

The results obtained for each object is very satisfying. After the first round of acquisition in natural lights, we were not able to adjust the cross of Clairmarais. The reflects visible on the images were too important and the image matching failed. In order to compute a model in such lightning condition, a manual correlation between images is necessary. The use of polarized lights overcomes this limitation and allows a perfect adjustment of each image. The results are presented in figure 11.

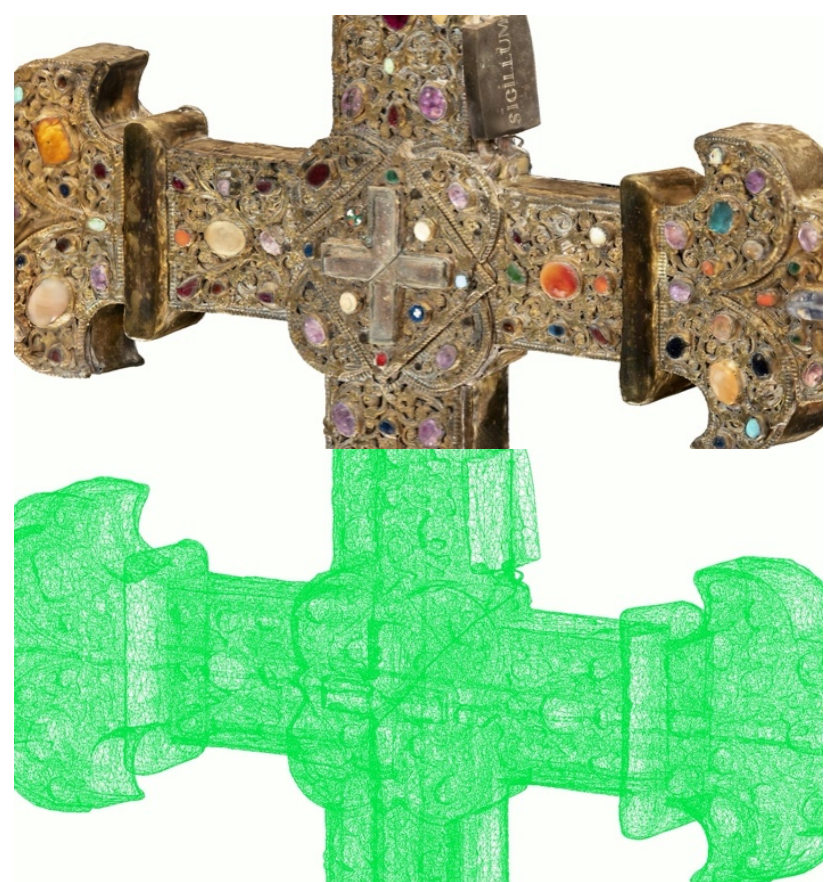

Figure 11. Final rendering of the Croix de Clairmarais based on corrected polarised light exposition. Textured model on the top and mesh on the bottom.

The pyxis was also computed using polarized light. In this case, the difficulty lies more in the high level of detail and the openings in the structure of the object. The large number of images acquired on a relatively small object $(12 \mathrm{~cm})$ allowed the model to be adjusted correctly The figure 12 shows the textured model and the calculated mesh.
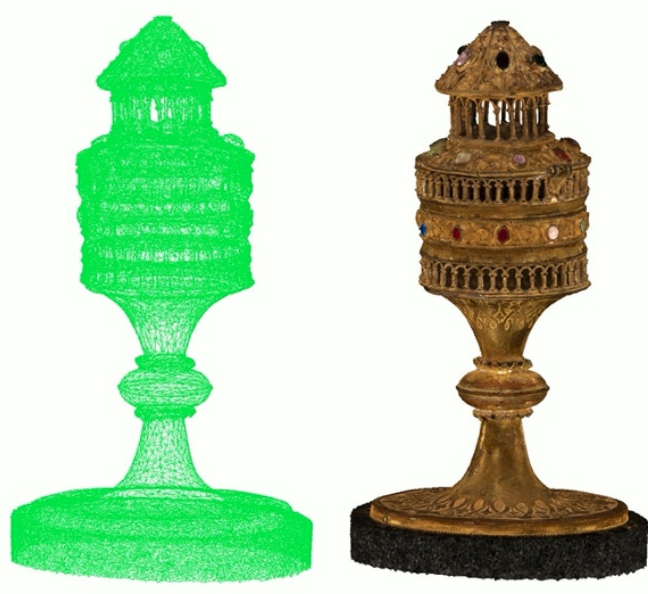

Figure 12. Final rendering of the Pyx based on corrected polarized light exposition. Textured model on the right and mesh on the left.

\subsection{Future developments}

As a future development, we plan to improve the colour correction methodology by testing several light configurations using a colour chart. In addition, on the basis of the current model, it is possible to perform a material segmentation of the model. This could be used as a basis for determining whether or not the colour change observed under polarized lightning conditions is systematic. If so, a model based on material correction can be established or the two different images (natural light and polarized light) can be used as a basis for segmentation. Finally, we plan to follow a different reconstruction methodology for the foot-cross. Indeed, to provide a coherent geometric representation and a faithful representation of the texture, the geometric model must be calculated on the polarized images and the texture extracted from the natural light images. The way to do this would be to take two pictures in the same state each time and replace the images in the adjustment processing chain for texture calculation. Another possibility is to calculate a cloud of points from the base of the object on polarized light and texture the cloud of points with the technology proposed in software such as Reality Capture. However, such a process is based on the similarity of images of coloured clouds and textures. This could be a limitation in the process. Given the application of mediation, a manual correction of the texture colour can be considered since the resolution of the usual mediation device is quite limited.

\section{HOLOGRAPHIC EXPLOITATION}

As explained above, e-thesaurus projects aim to provide mediation tools to present objects that are not easily transportable from museums. We have started a collaboration with Holusion (https://holusion.com/fr/) which develops holographic devices. The holograms presented are based on paper gloss technology. This technology is based on the reflection of an image on a glass oriented at 45 degrees from the users. The reflection of the images gives a "3D" representation floating in the air. To ensure a smooth representation of objects, 3D models must be simplified. Thanks to the calculation of several levels of detail, we were able to provide a simplified representation of the mesh size with a similar texture quality. The models have been implemented in the device and have participated in several exhibitions, one in Saint-Omer, France and the other in Liège, Belgium.

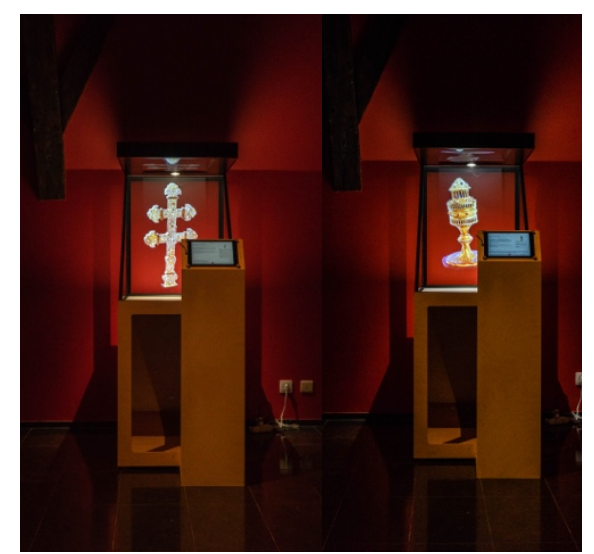

Figure 13. Holographic device rendering the cross of Clairmarais on the left and the pyxon the right.

The device is calibrated to provide a 1:1 scale representation of objects. This allows visitors to gain a better knowledge of the 
objects being observed. The development of some interactions with the model is planned in order to allow visitors to zoom or slice the model according to their wishes. The figure 13 shows the device in action.

\section{CONCLUSION}

The masterpieces of goldsmithing generally lack accessibility. This lack stems either from the public's ignorance of this type of art and the difficulty of transporting these objects for temporary exhibitions. The e-thesaurus projects aim to overcome these two limitations by providing support to researchers with a 3D modelling process of reflective and complex objects and a mediation result accessible to a wider audience. Two methodology of acquisition have been tested and combined in order to obtain the best possible result. Natural light illumination and cross-polarised lighting were tested on several pieces to evaluate the benefits and drawbacks of both methodologies. It appears that the cross-polarised light have an effect on the acquired colours. The colour modification seems to be related to the nature of acquired objects more than a global shift or modification of the image histogram, except a change of contrast. The proposed process helps at the information fusion in order to obtain a wider range of information for researches associated to the produced models.

\section{ACKNOWLEDGEMENTS}

The authors would like to thank the Musée de l'hôtel Sandelin (Saint Omer, France) for providing access of their preserved objects and materials. This research was carried out with the financial support of the MESHS, the CPER Mauve, the University of Liège and the University of Lille. The authors would like to thank all the e-thesaurus project partners for their advices and comments.

\section{REFERENCES}

Abate, D., 2019: Documentation of paintings restoration through photogrammetry and change detection algorithms. Heritage Science, 7(13). doi.org/10.1186/s40494-019-0257-y.

Conen, N., Hastedt, H., Kahmen, O., Luhmann, T., 2018: Improving image matching by reducing surface reflection using polarising filter techniques. Int. Arch. Photogramm. Remote Sens. Spatial Inf. Sci, II-2/W7, 267-274. doi.org/10.5194/isprsarchives-XLII-2-267-2018.

Crabb, P., 2001: The use of polarised light in photography of macrofossils. Palaeontology, 44, 659-664.

Edwards, N., 2011: Cross-Polarisation, Making it Practical. Journal of Visual Communication in Medecine, 34, 165-172. doi.org/10.3109/17453054.2011.635291

George, P., 2016: Orfèvrerie septentrionale L'oeuvre de la Meuse II, Archéoforum, Trésor de la Cathédrale de Liège, Liège.

Hallot, P., Gil, M., 2018 : e-thesaurus - Données liées à un modèle 3D de pièces d'orfèvrerie - modélisation sémantique et définition des besoins. In Moyen Age 2.0 Reconstructions Numériques et Histoire de l'art: Recherche et Communication. Université de Lausanne, Lausanne, Switzerland.

Hallot, P., Gil, M., Guillaumont, T., 2019: e.thesaurus : l'orfèvrerie à l'épreuve de la modélisation numérique - l'exemple de la valorisation du Pied de croix de l'abbaye de Saint-Bertin (Saint-Omer) sous forme holographique. In 144è Congrès National Des Sociétés Historiques et Scientifiques : Le Réel et Le Virtuel. Comité des travaux historiques et scientifiques, Marseille.

Luhmann, T., Robson, S., Kyle, S., Harley, I., 2007: Close range photogrammetry. Wiley.

Nicolae, C., Nocerino, E., Menna, F., Remondino, F., 2014: Photogrammetry applied to problematic artefacts. Int. Arch. Photogramm. Remote Sens. Spatial. Inf. Sci. V/23, 451-456.

Noya, N.C., Garcia, Á.L., Ramirez, F.C., García, Á.L., Ramírez, F.C., 2015: Combining photogrammetry and photographic enhancement techniques for the recording of megalithic art in north-west Iberia. Digital applications in archaeology and cultural heritage, 2(2-3), 89-101. doi.org/10.1016/j.daach.2015.02.004.

Wells, J., Jones, T., Danehy, P., Disease, I.B., 2005: Polarization and colour filtering applied to enhance photogrammetric measurements of reflective surfaces, 46th Structures, Structural Dynamics and Materials Conference. AIAA/ASME/ASCE/AHS/ASC, Austin, Texas p. 1887. doi.org/10.2514/6.2005-1887. 